$\begin{array}{cc}\text { TeLe }(\text { In })^{2} & \text { Contents lists available at ULERevistas } \\ \text { Innovation Institute } & \text { Teaching and Learning Innovation Journal } \\ \text { telein2.wordpress . com/ }\end{array}$

\title{
Del aula al museo: estrategias profesionalizantes de los alumnos de Historia del Arte de la Universidad de León a través de un proyecto de innovación docente (2017) From the class to the museum: professional strategies of students of Art History at the University of León through a project of teaching innovation (2017)
}

\author{
María Dolores Teijeira Pablos ${ }^{\mathrm{a}}$, Emilio Morais Vallejo ${ }^{\mathrm{a}}$, José Alberto Moráis Morán ${ }^{\mathrm{a}}$ \\ ${ }^{a}$ Departamento de Patrimonio Artístico y Documental, Universidad de León, Spain
}

\begin{abstract}
Este artículo aborda el desarrollo y los resultados de un proyecto de innovación docente aplicado en el grado de Historia del Arte de la Universidad de León, durante el año 2017, dentro del Plan de Innovación Docente de dicha institución. Se presentan los antecedentes de esta actividad, así como un breve estado de la cuestión sobre las publicaciones que abordaron el desempeño profesional de los estudiantes tras terminar sus estudios. Finalmente se explica la metodología del proyecto objeto de análisis, las fases de realización, su relación con las competencias propias de estos estudios y los resultados obtenidos.

Abstract- This article analyzes the development and results of a project of teaching innovation applied in the degree of History of Art of the University of León, during the year 2017, within the Plan of Teaching Innovation of this institution. The background of this activity is presented, as well as a brief state of the art on publications dealing with the professional performance of students after completing their studies. Finally, the methodology of the project under analysis, the phases of realization, its relationship with the competences of these studies and the results obtained are explained.
\end{abstract}

Keywords: Historia del Arte, innovación docente, profesionalización, museos

History of Art, teaching innovation, professionalization, museums

\section{Introducción}

La actividad docente precisa de una continua actualización e innovación para que sus resultados sean los más adecuados y los estudiantes puedan desarrollar su formación en relación con los requerimientos, siempre cambiantes, de la sociedad actual. Por ello las universidades españolas vienen desarrollando, desde hace ya aproximadamente dos décadas, estrategias para que su personal docente pueda incorporar en su actividad diaria las metodologías y recursos más innovadores. La Universidad de León convoca anualmente, desde 2013 como tal, aunque ya hubo experiencias anteriores con diferente formato y denominación, los Planes de Apoyo a la Innovación Docente (PAID),

Email addresses: md.teijeira@unileon.es (María Dolores Teijeira Pablos), emilio.morais@unileon.es (Emilio Morais Vallejo), jamorm@unileon.es (José Alberto Moráis Morán) en el marco de su Escuela de Formación del Personal Docente e Investigador, que pretende "fomentar la calidad y la innovación en la docencia" ${ }^{1}$.

En el marco de la convocatoria de 2017 se aprobó el proyecto Estrategias profesionalizantes del historiador del arte, en el que participaron varios profesores del Área de Historia del Arte del Departamento de Patrimonio Artístico y Documental ${ }^{2}$, que han ido desarrollando varias actividades dirigidas a proporcionar al alumno una primera toma de contacto con el mundo laboral dentro de su campo, entre ellas "Del Aula al Museo". I Plan de Colaboración entre la Universidad y los Museos Museos Catedralicio y

\footnotetext{
${ }^{1}$ Recuperado de: http://servicios.unileon.es/formacion-pdi/plande-apoyo-ala-innovacion-docente/, consultado el 12 de mayo de 2017.

${ }^{2}$ El mencionado proyecto se ha venido desarrollando durante el año 2017 por los profesores María Victoria Herráez Ortega, Roberto Castrillo, Joaquín García Nistal, Jorge Martínez Montero y los autores de esta comunicación Emilio Morais Vallejo, Jose Alberto Moráis Morán y María Dolores Teijeira.
} 
Diocesano de León, Museo de la Fundación Sierra Pambley y Museo de León, objeto de la presente comunicación ${ }^{3}$.

\section{Contexto}

A. La profesionalización del historiador del arte como objetivo de la innovación docente en España.

Como indicábamos en el inicio de este trabajo, a partir del establecimiento en España de los nuevos planes de estudio bajo las directrices del Espacio Europeo de Educación Superior (EEES) y dentro del Proceso de convergencia en un Estado Europeo de Educación superior, más conocido como "Proceso de Bolonia", la interacción del mundo laboral y profesional, con respecto a los nuevos titulados en historia del arte y al mismo "Plan Bolonia" ha sido tema de análisis en las universidades españolas ${ }^{4}$ (Martin, 2007).

En realidad, y desde una perspectiva nacional, estas experiencias realizadas tras el año 2000 tomaban como punto de partida otros hitos académicos llevados a cabo en la década de los 90 .

Sin pretender exhaustividad en el tema, la cuestión de la integración laboral y la importancia de la innovación docente ocupó algunas publicaciones, escasas, que vieron la luz por entonces, como la coordinada por Carlos Marcelo (Marcelo, 1996) $)^{5}$.

Dos años después veía la luz la publicación firmada por Ana María Alonso (1998), titulada El profesorado asturiano ante la enseñanza de la Historia del Arte, en el contexto de las que por entonces eran nuevas iniciativas en estas materias. Así, entre los días 17 y 19 de junio de 1992 se celebraron en Cádiz las Jornadas Historia del Arte y Bienes Culturales del Comité Español de Historia del Arte (CEHA), en colaboración con el Instituto Andaluz del Patrimonio Histórico, dependiente de la Consejería de Cultura de la Junta de Andalucía, que no fueron publicadas hasta 1998. El profesor Gonzalo M. Borrás Gualis desarrolló una ponencia, pionera, titulada "Fundamentos para la profesionalización de la Historia del Arte en España" (Borrás, 1998).

Podemos considerar estos antecedentes como referenciales, tanto para las reflexiones más actuales en torno al papel del historiador del arte frente al mundo profesional, como marco para comprender las acciones que a partir del

${ }^{3}$ En dicho proyecto, además de la actividad aquí analizada se ha desarrollado una segunda, bajo el nombre "El hall transformado", que recoge una experiencia que se desarrolla anualmente en la Facultad de Filosofía y Letras durante la primavera de cada curso y que recoge una obra performativa -instalación- de un artista en cuyo montaje, exhibición y análisis participan los alumnos de la titulación de Historia del Arte.

${ }^{4}$ Por ejemplo, en Valencia, en el año 2006, se realizaron las Jornadas de Historia del Arte y Espacio Europeo de Educación Superior que dio lugar a una publicación que ya por entonces valoraba el impacto de los nuevos planes en la incorporación laboral de los egresados.

${ }^{5}$ Es muy significativo cómo aún en dicha publicación no se incluía un apartado específico para la disciplina. año 2000 realizaron en España diferentes universidades y grupos de innovación docente.

No sólo han proliferado publicaciones sobre el tema, proyectos de investigación e innovación aplicados a la docencia y al mundo laboral. Ya en fechas más recientes se ha creado, desde la Universidad de Oviedo, el Instituto de Investigación e Innovación Educativa (INIE) ${ }^{6}$, claro reflejo del interés y necesidad de combinar estos factores a favor de una mejor incorporación de los egresados a la vida laboral.

Tales experiencias docentes fueron acompañadas de la publicación de diversos artículos indexados en revistas de impacto y actas de congreso así como del volcado de la información en repositorios digitales, siendo estas tres vías las más usuales en la difusión de los procesos de investigación y los resultados derivados de los proyectos de innovación docente que tuvieron como objetivo el desarrollo de competencias dirigidas a acercar a los alumnos al ámbito laboral.

Desde la Universidad de Santiago de Compostela se han organizado diversas acciones dirigidas a valorar las nuevas estrategias de innovación docente en el campo de las humanidades, con especial interés por la historia del arte, dando lugar a la publicación del año 2011 titulada Innovación metodológica y docente en historia, arte y geografía ${ }^{7}$.

Igualmente, desde la Universidad Complutense de Madrid son meritorios los trabajos del Grupo de Innovación Docente del Departamento de Historia del Arte I (Medieval), que ha centrado sus investigaciones y aportes en materia de innovación docente sobre iconografía medieval, campo generalmente olvidado con respecto a su relación con las nuevas tecnologías y la indexación de corpus digitales, y que se ha convertido en una herramienta esencial en los dominios web ${ }^{8}$ (González y otros, 2012).

Finalmente y también en la Universidad Complutense de Madrid, en el año 2015 se llevó a cabo otro proyecto

${ }^{6}$ Dirigido en el año 2017 por Emilio Álvarez Arregui, siendo una de las funciones básicas del Instituto: "Asesorar y orientar a los profesionales que desempeñan sus funciones en los ámbitos educativo y de la formación de manera que se aporte la valoración científica necesaria, se sugieran las recomendaciones oportunas, se faciliten los seguimientos y se favorezca la metaevaluación con el fin de mejorar el diseño, la planificación, la implementación, la evaluación, los resultados, la transferencia y el impacto", recuperado de: https://inie.uniovi.es/presentacion/objetivos, consultado el 1 de mayo de 2017.

${ }^{7}$ Dentro de este volumen, resulta significativa la investigación de Cruz Yábar (2012), por las vinculaciones con el proyecto que estamos realizando desde el Departamento de Patrimonio Artístico y Documental de la Universidad de León. En el mismo volumen se publicó el trabajo que consideramos un precedente para las nuevas perspectivas investigadoras que desarrollamos en el año 2017 de Flórez, Teijeira y Campos (2012).

${ }^{8}$ Fruto de diversos proyectos de esta índole realizados entre los años 2009 y 2016 y dirigidos por Irene González Hernando, se ha gestado la Base de Datos Digital de Iconografía Medieval (I, II, III, IV, V y VI parte) y se publica, desde 2009, la Revista Digital de Iconografía Medieval, dirigida igualmente por la Dra. González Hernando. 
que tuvo como objetivo valorar las oportunidades laborales de los nuevos historiadores del arte, convirtiéndose esta institución en una de las más señeras a nivel nacional en fomentar esta vía de trabajo ${ }^{9}$.

Para terminar esta contextualización de los objetivos que guían el trabajo del Grupo de Innovación Docente de la Universidad de León y las actividades desarrolladas en el PAID del año 2017, con respecto a las experiencias de otros centros e instituciones, resultan relevantes los proyectos desarrollados desde la Universidad Rovira i Vigilli (Tarragona) y coordinados por Marta Serrano Coll entre los años 2012 y $2017^{10}$.

Otro capítulo relevante en relación con la aplicación de los procesos de aprendizaje desarrollados en el aula sobre los ámbitos profesionales recae sobre los museos.

Tanto los espacios expositivos más tradicionales como los nuevos centros de creación y exhibición artística se han convertido en los lugares por excelencia donde los historiadores del arte han encontrado un óptimo nicho de "profesionalización". Así mismo, también son estos espacios los que con mayor frecuencia se mencionan en las guías docentes, a la hora de concretar la aplicación práctica de las capacidades y competencias desarrolladas en la docencia universitaria.

Nuevamente la bibliografía existente sobre el tema es amplia. Los clásicos trabajos realizados por Greenberg (1996) y Hooper-Greenhill (1999a, 1999b, 2007) han enmarcado la práctica museística en el contexto de la enseñanza tanto en los niveles iniciales como desde la Universidad. Ello ha tenido su eco sobre otras propuestas desde la investigación española (Coca y Pérez, 2011).

Por otra parte, también la historiografía española inició tempranamente esta vía de trabajo, en los primeros años de la década de los 90. En tales fechas, por ejemplo, Delmé, Forrelland, Gratacós y Oliver (1993) publicaron un estudio sobre el papel de las obras plásticas en los procesos de aprendizaje y evaluación.

\footnotetext{
${ }^{9}$ Proyecto de Innovación y Mejora de la Calidad Docente Convocatoria 2015. Educación, investigación y oportunidades laborales. Talleres de introducción a la práctica laboral en el campo de la Historia del Arte y la Arqueología, coordinado por Daniel Ortiz Pradas, Facultad de Geografía e Historia Departamento de Historia del Arte I (Medieval), recuperado de: http://eprints.ucm.es/35039/1/Memoria_Final_Proyecto_PIMCD_367.pdf, consultado el 29 de abril de 2017. Con idéntico interés en la inserción laboral de los alumnos de historia del arte, se realizó, un año antes, el proyecto titulado "Proyecto de Innovación y Mejora de la Calidad Docente Convocatoria 2014. Educación, investigación, nuevas tecnologías y oportunidades laborales: talleres de iniciación a las metodologías prácticas en el campo de la Arqueología y de la Historia del Arte antiguo", coordinado por Jorge García Sánchez Facultad de Geografía e Historia, Ciencias y Técnicas Historiográficas y de Arqueología, recuperado de: https://eprints.ucm.es/28147/3/PIMCD $\% 202014 \% 20$ n $\%$ C2\%BA\%2059.pdf, consultado el 12 de abril de 2017.

${ }^{10}$ Son importantes las Jornadas del año 2012, tituladas "El Rol actual de la Historia del Arte (I): Del purgatorio académico al compromiso social" y las de 2013, que versaron sobre "El Rol actual de la Historia del Arte (II): Visiones periféricas de una profesión".
}

A partir del año 2000 los museos se convirtieron en los espacios de investigación sobre la practicidad de la teoría propia de la historia del arte, destacando, por ejemplo, los trabajos de Rico, Ávila (2004), Rivero (2009), Fontal (1999, 2004, 2009). Citamos igualmente la línea de trabajo de Esther Lozano (2017), que viene desarrollando propuestas de innovación docente planteadas para los alumnos, remarcando el potencial educativo de la Historia del Arte y los museos en el futuro profesional de los estudiantes universitarios dedicados a la docencia ${ }^{11}$.

Finalmente nos parecen relevantes los dos volúmenes monográficos dedicados al tema. El primero, el de la revista de innovación e investigación educativa Tarbiya, del año 2009, que se dedicó a la didáctica de las Ciencias Sociales y la educación en museos. Dicha publicación compendió algunas aportaciones donde la problemática de las posibilidades profesionales del historiador del arte se convirtieron en el eje de algunos estudios (Blanco y de los Reyes, 2009).

En segundo lugar merece mencionarse el trabajo conjunto en la revista Patrimonio y Educación del Instituto de Patrimonio Cultural de España (Fontal, 2011 y Querol, 2011).

Siempre en el caso español y a partir de los precedentes citados, la investigación y la innovación docente se unieron para generar interesantes proyectos, como el realizado en la Universidad Complutense de Madrid y que se tituló "Descubriendo la bioquímica en las colecciones de arte del Museo del Prado" ${ }^{12}$. El proyecto, con clara vocación interdisciplinar e interdepartamental, reunió a docentes del Departamento de Bioquímica y Biología Molecular I y el Departamento de Historia del Arte II (Moderno) a partir de una colaboración con el Museo del Prado. La metodología del Aprendizaje Basado en Problemas (ABP) buscó siempre "nuevas perspectivas para la futura inserción laboral" ${ }^{13}$, mostrando en todo caso las nuevas directrices en las que se enmarcan hoy los proyectos de innovación docente en los que la historia del arte y los museos toman un protagonismo vital en la búsqueda de nuevas perspectivas laborales para los estudiantes y egresados.

\section{B. La experiencia previa del grupo de trabajo}

En este contexto varios de los profesores del Área de Historia del Arte de la ULE hemos querido trabajar con nuestros alumnos desarrollando un proyecto que les permita una primera toma de contacto con el mundo laboral de la historia del arte. De este modo "Del Aula al Museo" surgió como una iniciativa del Departamento de Patrimonio Artístico y Documental y del Instituto de Estudios

\footnotetext{
${ }^{11}$ La autora utiliza una metodología de aprendizaje que aúna el estudio de casos y el role-playing con el objetivo de recrear una realidad profesional vinculada a los museos.

${ }^{12}$ Proyecto de Innovación y Mejora de la Calidad Docente número 64 del Vicerrectorado de Evaluación de la Calidad, Universidad Complutense de Madrid, Convocatoria 2013.

${ }^{13}$ Véase un resumen del citado proyecto recuperado en: http://rib.bioucm.es/cont/docus/innovacion/2-2015-06-08Museo\%20del\%20Prado.pdf, consultado el 3 de junio de 2017.
} 
Medievales de la Universidad de León para materializar los deseos de colaboración de estos centros con los museos leoneses, ya iniciada anteriormente, pero que quería ser consolidada por ambas partes, y la búsqueda de nuevas perspectivas laborales para los alumnos del Grado de Historia del Arte de dicha Universidad.

El establecimiento de contactos entre el estudiante universitario y el mundo laboral durante el periodo de formación del primero es un paso de enorme importancia para su futura integración profesional, sea cual fuere su puesto y centro de trabajo futuro. Por esta razón, y conociendo las dificultades de empleabilidad de algunos estudios, especialmente en la rama de Artes y Humanidades , en ocasiones por el propio desconocimiento del alumno de sus posibilidades laborales, en el Grado de Historia del Arte de la Universidad de León hemos intentado, ya desde la implantación de la Licenciatura en Historia del Arte, mostrar a nuestros estudiantes, de la manera más directa posible, el amplio abanico de opciones para el desarrollo de su actividad profesional una vez obtenido su título oficial. Para ello hemos puesto en marcha diferentes actividades docentes, además de fomentar aquellas otras que ya se desarrollan a nivel oficial con el mismo fin, o similar, como por ejemplo las prácticas externas en empresas, las salidas de campo o las prácticas fuera del aula.

Previamente a la puesta en marcha del proyecto Estrategias profesionalizantes del historiador del arte, se desarrollaron, en el marco del Departamento de Patrimonio Artístico y Documental y también como proyectos del correspondiente Plan de Apoyo a la Innovación Docente de la ULE, los titulados "La integración del sector profesional en las prácticas de Historia del Arte", en sendos cursos consecutivos 2008-2009 y 2009-2010, coordinados por la Dra. Flórez Crespo y con la colaboración en la organización de las Dras. Campos Sánchez-Bordona y Teijeira Pablos, del mencionado departamento.

El proyecto se centró en la celebración de unas Jornadas Prácticas, con un desarrollo similar al del taller (workshop), en el que los alumnos de la entonces licenciatura en Historia del Arte pudieron contar con la contribución de profesionales de los campos de la gestión cultural, a nivel nacional y autonómico, del mundo de los museos y de la restauración de bienes culturales. Con ellos pudieron trabajar en diez talleres diferentes sobre temas relacionados con la adquisición, protección, conservación e intervención de bienes culturales, conociendo sus competencias profesionales, las labores desarrolladas en su trabajo y la aplicación de los conocimientos del historiador del arte en ellas. Los conocimientos y competencias adquiridos durante las talleres, que se desarrollaron en la Facultad de Filosofía y Letras, se completaron con visitas a algunos de los centros dónde estos profesionales realizaban su labor u otros similares; de este modo los alumnos pudieron conocer de cerca la actividad del Centro de Conservación y Restauración de Bienes Culturales de Castilla y León, en Simancas (Valladolid), el Instituto del Patrimonio Histórico Español (hoy Instituto del Patrimonio
Cultural de España), en Madrid, y la National Gallery de Londres. Los contactos establecidos durante estas Jornadas sirvieron también para perpetuar en el tiempo la posibilidad de visitar estos centros para futuros alumnos, pudiendo contar nuestros estudiantes, hasta hoy, con el conocimiento directo de su funcionamiento a través del testimonio de sus trabajadores, muchos de los cuales proceden, como los estudiantes, de la formación en historia del arte.

Esta experiencia previa, de resultados enormemente positivos y muy bien valorada por los alumnos, nos ha ayudado, y animado, a organizar el presente proyecto, pensando en lo que la actividad universitaria, especialmente la de los estudiantes, puede aportar a los museos, como centros de investigación y protección de las obras de arte objeto de estudio del historiador del arte, y lo que los museos pueden enseñar a nuestros alumnos como centros en los que se desarrolla una parte importante de la labor profesional del historiador del arte.

\section{Descripción}

La actividad formativa diseñada con el título "Del aula al museo" posee un sentido práctico orientado a enseñar a los alumnos cómo deben actuar para que después ellos apliquen lo aprendido, pudiendo actuar por sí mismos.

Fue planificada en tres fases sucesivas, teniendo cada una de ellas unos objetivos claros con la intención de ir avanzando progresivamente en el proceso de aprendizaje del alumno, tanto inducido como autónomo, fomentando de esta manera el autoaprendizaje, entendido como una faceta fundamental que debe facilitar el profesor. Además, siempre se trató de que los alumnos al tiempo que adquirían competencias genéricas o transversales, por un lado, y competencias específicas de la titulación de Historia del Arte, por otro.

Participaron tres museos de León, el Museo Catedralicio y Diocesano de León, el Museo de la Fundación Sierra Pambley y el Museo de León, que previamente fueron contactados y mostraron desde el primer momento su disposición para colaborar; de no haber sido así, la actividad hubiera sido totalmente inviable, pues es necesaria la implicación directa de algún responsable de la institución para llegar a buen fin ${ }^{14}$. Las asignaturas implicadas fueron Arte Gótico Español de $3^{\circ}$ curso, Artes Suntuarias y Diseño Industrial de $4^{\circ}$ curso y Arte Barroco Español de $4^{\mathrm{o}}$ curso, todas ellas del Grado de Historia del Arte. Cada asignatura orientó su práctica hacia distintas etapas de la Historia y se fijó para ello en diferentes museos, teniendo en cuenta la mejor adaptación de cada fondo museístico

\footnotetext{
${ }^{14}$ Agradecemos la colaboración desinteresada y entusiasta de Patricia Centeno, responsable del Museo de la Fundación Sierra Pambley, Jairo Fernández Estrada, responsable del Departamento de Educación y Acción Cultural (DEAC) del Museo de León y Luis Grau Lobo, director del mismo, y de Máximo Gómez Rascón, director del Museo Catedralicio y Diocesano de León.
} 
a los objetivos de la materia de estudio. Los alumnos de Arte Gótico podían estudiar una pieza de este periodo en el Museo de la Catedral o en el Museo de León, los de Artes Suntuarias en el Museo Sierra Pambley y los de Barroco Español en el Museo de León.

La primera fase consistió en la presentación de la práctica a los alumnos por parte del profesor de la asignatura en el aula, con la intención de motivarlos. A continuación los alumnos de las asignaturas implicadas realizaron una visita guiada a cada uno de los museos con el fin de que conocieran las posibles piezas de estudio mediante una explicación somera realizada por personal del museo. Esta mirada general hace que los alumnos se fijen con más detenimiento en las obras de arte expuestas, pues, mientras las están contemplando con una visión crítica, van recordando los conceptos adquiridos en el desarrollo de la asignatura y estableciendo un cotejo entre sus conocimientos y sus gustos para elegir la pieza que estudiarán más tarde. Es interesante que en esta fase pueda desarrollarse la enseñanza emocional que surge de la comunicación directa con la obra de arte y con los comentarios e interrelaciones que se suscitan entre los estudiantes. Para finalizar este apartado cada alumno eligió la pieza que quería estudiar según sus propios intereses, por lo tanto sin ninguna orientación dirigida por el profesor.

En la segunda fase los alumnos se dedicaron a revisar la bibliografía existente sobre la obra escogida y procedieron a su estudio, lo que llevó a visitar, entonces ya individualmente, la pieza seleccionada. También buscaron en el archivo del museo toda la información disponible sobre la pieza; de esta manera conocieron el museo desde dentro, algo novedoso para ellos, y se familiarizaron con las herramientas y recursos museísticos, por ejemplo el sistema integrado de documentación y gestión museográfica DOMUS. Con el material recogido elaboraron fichas para realizar un dossier que les facilitara posteriormente la redacción del texto. Es de especial interés en este momento la aplicación de las técnicas propias del aprendizaje de la Historia del Arte y el desarrollo de una incipiente tarea de investigación. Para finalizar esta parte, los alumnos redactaron un texto en el que estaban obligados a utilizar el sistema de citas bibliográficas a pie de página que tiene normalizado el Grado de Historia del Arte, así como la elaboración del correspondiente anexo documental. Una vez terminado este trabajo y entregado en un cuadernillo impreso, fue corregido y calificado por el profesor y por el responsable del museo correspondiente, quienes seleccionaron los mejores para que fueran expuestos al público delante de la pieza analizada.

La tercera fase consistió en la exposición del trabajo en el museo, ante el público asistente en el Día Internacional de los Museos y los técnicos del museo, buscando que la experiencia fuera más enriquecedora al poder ofrecer observaciones directas de los especialistas. Para preparar la explicación pública, los alumnos elaboraron una "hoja de sala" en la que resumían su trabajo y lo adaptaban desde unos parámetros académicos a otros más divulgativos. En esta hoja se recoge la información esencial y suficiente para que los visitantes puedan entender la obra de arte en caso de contemplarla sin la ayuda de la explicación de un guía. Esta "hoja de sala" queda a disposición del museo para que la consulten los futuros visitantes y se solicita que sea incorporada a la página web del museo para dejar constancia de la labor realizada, sirviendo al mismo tiempo para que los alumnos se sientan más satisfechos del esfuerzo realizado.

La actividad se evaluó del mismo modo que el resto de actividades formativas que los alumnos deben realizar dentro de sus correspondientes asignaturas, realizando los estudiantes que optaron por no participar otras tareas similares en su lugar.

El desarrollo de esta actividad carecería de valor si no sirviera para la consecución de una serie de competencias genéricas y específicas asociadas al Título de Grado en Historia del Arte, que aparecen recogidas en el Real Decreto $1393 / 2007$.

Entre las competencias genéricas o transversales, comunes a cualquier titulación, con este ejercicio los alumnos adquieren la capacidad de tomar decisiones, de diseñar proyectos, de relacionarse socialmente, de gestionar la totalidad de una actuación y la búsqueda y selección de información de la misma, y de adquirir autonomía en el aprendizaje de nuevos conocimientos y técnicas, en muchos casos averiguadas por ellos.

Al tener que actuar ante un público desconocido, los alumnos se preparan para desarrollar el dominio de la expresión oral, y, en su caso, el probable uso de una lengua extranjera.

En cuanto a las competencias específicas, adquieren habilidades propias o vinculadas a la Historia del Arte y se acercan al conocimiento de las posibilidades de trabajo futuro. En este sentido podemos decir que son también "profesionalizantes". Si las distintas materias impartidas en la titulación tienen como objetivo saber, saber hacer y saber ser, creemos que con esta actividad las tres posibilidades se acrecientan al realizar una tarea que el alumno asume como reto personal.

$\mathrm{Al}$ enfrentarse directamente con una obra de arte desarrollamos el espíritu crítico del alumno, su sensibilidad para verla y aumentar su capacidad para apreciar los valores estéticos intrínsecos en ella.

Como deben utilizar una metodología científica y unas herramientas específicas de la Historia del Arte, amplían las posibilidades de análisis e interpretación de las fuentes propias de la disciplina.

Por otro lado, el estudio sistemático de las obras de arte exige al alumno la adquisición de conciencia de la necesidad de formarse en diversos campos interdisciplinares, aumentando sus conocimientos y las posibles relaciones que existen realmente entre diferentes disciplinas.

Al tener que utilizar bibliografía y documentación variada, adquieren conciencia crítica de las coordenadas espaciotemporales y de los límites e interrelaciones geográficas y culturales de la Historia del Arte. 
Al mismo tiempo se acercan al conocimiento de las distintas metodologías de la Historia del Arte y de su contingencia histórica (Historiografía del Arte).

Por las características propias de la actividad descrita, necesariamente los alumnos tienen que conocer y ampliar la terminología precisa y específica de la Historia del Arte.

Cierra este apartado competencial una capacidad que engloba a la mayoría de las señaladas más arriba. El alumno adquiere un conocimiento sistemático e integrado del hecho artístico, comprobando distintos lenguajes, procedimientos y técnicas de la producción artística que ha habido a lo largo de la Historia. E incluso, conocimiento de la teoría y pensamiento estético.

En cuanto a competencias "profesionalizantes", la actividad ofrece la posibilidad de adquirir un conocimiento práctico de los procesos básicos de la metodología científica en la Historia del Arte, como pueden ser el estado de la cuestión, análisis integral de la obra de arte, búsqueda de la información inédita, planteamiento de hipótesis, procesos críticos de síntesis, obtención de conclusiones, crítica del Arte, etc.

Al trabajar con obras que están custodiadas en un museo, les permite conocer los problemas que plantean actualmente la conservación, restauración y gestión del Patrimonio Artístico y Cultural.

El trato con los especialistas encargados de los museos les dan conocimientos básicos de museología y museografía, al tiempo que conocen posibles salidas profesionales tras la terminación de los estudios universitarios.

También destacamos la adquisición de conocimientos sobre gestión de colecciones de arte, entre las que podemos destacar el inventario, documentación, catalogación, montaje de exposiciones y la difusión del patrimonio.

\section{Resultados}

Como conclusión podemos afirmar que los resultados de la experiencia fueron positivos para los alumnos, que conocieron de primera mano el funcionamiento de los museos, mantuvieron enriquecedoras relaciones con el personal técnico de los mismos y se acercaron al mundo laboral en el que probablemente tengan posibilidad de futuro. Pero también para los propios museos que descubrieron otra manera de colaborar con la universidad, acusaron algunas carencias que resaltaron los estudios de los alumnos y encontraron una forma diferente de llegar al público. Estos aprendizajes de doble sentido siempre resultan enriquecedores para los implicados.

Los satisfactorios resultados del I Plan, que se plasmaron en las encuestas que público y estudiantes cumplimentaron a su finalización, así como la opinión favorable de los responsables de los museos implicados, nos han animado a plantear la continuidad del Plan en futuros cursos, sirviendo además como base de actuación para el Grupo de Innovación Docente (GID) id-Arte, de momento grupo de trabajo, pero que esperamos se convierta en grupo reconocido por la Universidad de León en breve plazo.
Esta impresión positiva, sin embargo, no supone una mera complacencia en lo ya realizado, sino un estímulo para mejorar el Plan en futuras convocatorias, incluyendo quizá más centros y alumnos, planteándolo dentro de un planteamiento más ambicioso que pueda, por ejemplo, incluir los Trabajos Fin de Grado y Fin de Máster de nuestros alumnos, en relación con los planes de investigación de los propios museos y buscando nuevas vías de difusión de la actividad para así poder llegar a un público aún más amplio.

\section{Referencias}

[1] Alonso Gutiérrez, A. M. (1998) El profesorado asturiano ante la enseñanza de la Historia del Arte, Oviedo, España: Universidad de Oviedo, Servicio de Publicaciones.

[2] Blanco Jiménez, C. y Reyes Leoz, J. L. de los (2009). Introducción. Universidad, museos y centros de enseñanza: un espacio educativo compartido. Tarbiya 40: 5-14.

[3] Coca Jimenez, P., y Pérez Mulas, A. (2011). Repensar las prácticas educativas en museos y centros de enseñanza Relato de una experiencia. Arte y políticas de identidad 5: 13-26.

[4] Cruz Yábar, M. T. (2012). Organizando una exposición: experiencia e innovación en la metodología docente de la asignatura de máster Gestión y planificación de museos y de las exposiciones temporales y su aplicación a la de Museología y Museografía del grado en Historia del Arte. En Durán Villa, F. R., López Facal, R., Saavedra Vázquez, M. del, Sánchez García, J. A., Villarino Pérez, M. (coords.). (2012). Innovación metodológica y docente en historia, arte y geografía. Actas Congreso Internacional, Santiago de Compostela, 7-9 de septiembre de 2011. Santiago de Compostela, España: Universidade de Santiago de Compostela, Servizo de Publicacións e Intercambio Científico, 1081-1090.

[5] Delmé, D., Forrellad, M., Gratacós, R., y Oliver, M. (1993). Educación artística: plástica. En Los procedimientos: aprendizaje, enseñanza y evaluación. Barcelona, España: ICE UB, 1993, 87-118.

[6] Durán Villa, F. R., López Facal, R., Saavedra Vázquez, M. del, Sánchez García, J. A., Villarino Pérez, M. (coords.). (2012). Innovación metodológica y docente en historia, arte y geografía. Actas Congreso Internacional, Santiago de Compostela, 7-9 de septiembre de 2011. Santiago de Compostela, España: Universidade de Santiago de Compostela, Servizo de Publicacións e Intercambio Científico.

[7] Fontal, O. (1999a). Historia: análisis del pasado y proyecto social. Barcelona, España: Editorial Crítica.

[8] Fontal, O. (1999b). Los museos de arte: un campo emergente de investigación e innovación para la enseñanza del arte. REIFOP 12 (4): 75-88.

[9] Fontal, O. (2004). La dimensión contemporánea de la cultura. Nuevos planteamientos para el patrimonio cultural y su educación. En Comunicación educativa del patrimonio: referentes, modelos y ejemplos. Gijón, España: Trea, pp. 81-104.

[10] Fontal, O. (2011). El patrimonio en el marco curricular español. Patrimonio y Educación 5: 21-44.

[11] Flórez Crespo, M. del M., Teijeira Pablos, M. D., Campos Sánchez-Bordona, M. D. (2012). La incorporación del ámbito profesional en los estudios de Historia del Arte. En Durán Villa, F. R., López Facal, R., Saavedra Vázquez, M. del, Sánchez García, J. A., Villarino Pérez, M. (coords.). (2012). Innovación metodológica y docente en historia, arte y geografía. Actas Congreso Internacional, Santiago de Compostela, 7-9 de septiembre de 2011. Santiago de Compostela, España: Universidade de Santiago de Compostela, Servizo de Publicacións e Intercambio Científico, pp. 1097-1112. 
[12] González Hernando, I., Fernández Fernández, L., Asís García García, F. de, Manzarbeitia Valle, S., Poza Yagüe, M., Rodríguez Peinado, L., Walker Vadillo, M. (2012). Un proyecto de innovación docente para mejorar la docencia de la iconografía medieval. En Durán Villa, F. R., López Facal, R., Saavedra Vázquez, M. del, Sánchez García, J. A., Villarino Pérez, M. (coords.). (2012). Innovación metodológica y docente en historia, arte y geografía. Actas Congreso Internacional, Santiago de Compostela, 7-9 de septiembre de 2011. Santiago de Compostela, España: Universidade de Santiago de Compostela, Servizo de Publicacións e Intercambio Científico, pp. 1128-1138.

[13] Greenberg, R., Ferguson, B. y Nairne, S. (1996). Thinking about exhibitions. London, Reino Unido: Routledge.

[14] Hooper-Greenhill, E. (1999a). The educational role of the museum. London, Reino Unido: Routledge.

[15] Hooper-Greenhill, E. (2007). Museum and education purpose pedagogy performance. New York, Estados Unidos: Routledge.

[16] Hooper-Greenhill, E. (1999b). Museums and Interpretive Communities. En Musing on Learning Seminar, Australian Museum. 20, recuperado en: australianmuseum.net.au/Uploads/Documents/2004/paper2.pdf, consultado el 5 de junio de 2017.

[17] Lozano, E. (2017). Patrimonio, Arte y Didáctica de las Ciencias Sociales. Análisis y reflexiones sobre una estrategia de aprendizaje en el marco de innovación docente. Clío 39, recuperado en http://clio.rediris.es, consultado el 15 de abril de 2017.

[18] Marcelo García, C. (coord..) (1996). Innovación educativa, asesoramiento y desarrollo profesional. Madrid, España: Ministerio de Educación y Ciencia.

[19] Martín Martínez, J. (2007). ¿Para qué estudiar historia del arte en la Universidad? Por unas Humanidades visuales. Ars Longa 16: 143-174.

[20] Querol, M. de los (2011). El patrimonio cultural en las universidades españolas: no sólo una cuestión de tiempo. Patrimonio y Educación 5: 75-80.

[21] Rico, L., Ávila, R. M. (2004). Los museos virtuales: nuevos ámbitos para aprender a enseñar el patrimonio históricoartístico: una experiencia en la formación de maestros. En Formación para la ciudadanía: las TICs y los nuevos problemas. Alicante, España: Asociación Universitaria de Profesores de Didáctica de las Ciencias Sociales, $1-16$.

[22] Rivero, M. P. (2009). Museos y didáctica on line: cinco ejemplos de buenas prácticas. Hermes: Revista de museología 1: 110-114. 